\title{
Cannabidiol and mental health: possibilities, uncertainties, and controversies for addiction treatment
}

\author{
Felix H. Kessler, ${ }^{1,2}$ (D) Lisia von Diemen, ${ }^{1,2}$ (iD Felipe Ornell, ${ }^{1,2}$ (iD Anne O. Sordi ${ }^{1}$ (iD \\ ${ }^{1}$ Centro de Pesquisa em Álcool e Drogas, Hospital de Clínicas de Porto Alegre (HCPA), Universidade Federal do Rio Grande do Sul (UFRGS), \\ Porto Alegre, RS, Brazil. ${ }^{2}$ Programa de Pós-Graduação em Psiquiatria e Ciências do Comportamento, UFRGS, Porto Alegre, RS, Brazil.
}

Interest in cannabidiol (CBD) is not recent, and its use and effectiveness have been controversial. On the one hand, therapeutic potential has been used as a false argument for legalization of growing and smoking marijuana, with all its components. On the other hand, the addictive potential of delta-9-tetrahydro-cannabinol (THC) has generated prejudice from the scientific community in studying the pharmacological properties of this specific molecule (CBD). In the last decades of the 20th century, however, the discovery of the endogenous endocannabinoid system and the isolation of CBD in the laboratory made it possible to conduct methodologically robust investigations on the therapeutic potential of CBD. Curiously, one of the promising perspectives is precisely in the therapy of addictions. ${ }^{1-3}$

In the psychiatric field, cannabis - which is still the most frequently used illicit psychoactive substance in the world - is usually associated with harmful effects, mainly related to addictive effects and psychiatric symptoms such as anxiety, depression, or psychosis. Cannabis sativa contains many other cannabinoids and terpenes, with CBD and THC being the two most studied. CBD has excelled as a potential pharmacological tool for organic and mental disorders. It is relevant to note that CBD and THC have very distinct biological effects. While THC has primarily psychoactive effects and is associated with cognitive deficits, CBD usually has none of these effects and may even attenuate them. ${ }^{4,5}$ The anticonvulsant effects of CBD were the first to be demonstrated as pharmaceutically useful. Currently, CBD is approved by the U.S. Food and Drug Administration to treat seizures caused by the Dravet and Lennox-Gastaut syndromes. The therapeutic effects of CBD are still being explored in clinical trials for a variety of diseases, such as Parkinson disease, breast cancer, dementia, ulcerative colitis, and many others. ${ }^{5,6}$

The mechanisms of action of CBD are still not completely elucidated. While THC acts as an agonist at cannabinoid receptors, CBD seems to have a more antagonist effect and interacts with other neurotransmitter receptors, such as serotonin, opioid, and dopamine. ${ }^{7}$ It appears to act by decreasing the reuptake of anandamide,

Correspondence: Felix H.P. Kessler, Centro de Pesquisa em Álcool e Drogas, Hospital de Clínicas de Porto Alegre, Universidade Federal do Rio Grande do Sul, Rua Ramiro Barcelos, 2350, Santa Cecília, Porto Alegre, RS, Brazil.

E-mail: fkessler@hcpa.edu.br

Submitted Feb 27 2021, accepted Apr 05 2021, Epub May 172021. an endocannabinoid produced by humans. CBD receptors are spread throughout many brain areas, as well as other parts of the body, including the gastrointestinal tract and immune system. In the brain, studies reveal that CBD acts on the medial prefrontal cortex (mPFC), amygdala, and hippocampus, which are areas directly related to the reward system. ${ }^{8}$ Therefore, it is reasonable to explore how CBD can be a potential treatment target for addiction disorders.

Regarding the role of CBD as a potential pharmaceutical agent in psychiatric disorders, studies have demonstrated its anxiolytic, antidepressant, and antipsychotic properties. Most of these studies were conducted with animal models and showed that lower doses of CBD $(10-20 \mathrm{mg} / \mathrm{kg})$ could decrease anxiety symptoms, but higher doses could not produce the same effects, suggesting a bell-shaped dose-response curve. ${ }^{9}$ Also, most of the studies were designed to test the effectiveness of CBD as an anxiolytic agent before a stressful situation. Nevertheless, there are no data to prove its effectiveness with a longer follow-up. ${ }^{10}$

In the addiction field, there is a need for new pharmacological tools to minimize symptoms of substancerelated disorders and prevent drug relapse. The anxiolytic effects of CBD were first demonstrated by its use as an agent that could attenuate the anxiogenic effects of $\mathrm{THC}$. Later, a few clinical trials using CBD for the treatment of heroin withdrawal showed its potential in decreasing craving symptoms. Studies have also begun to elucidate the effects of CBD on drug memory expression using paradigms with translational relevance to addiction. Some findings suggest that CBD reduces the expression of drug memories acutely and by disrupting their reconsolidation. There is hope that understanding how CBD regulates emotion and emotional memory processing may eventually lead to its use as a treatment for substance abuse. ${ }^{11}$

As noted in a recent review, preliminary, preclinical results suggest that CBD can attenuate alcohol consumption and potentially protect against specific harmful effects of alcohol, such as liver and brain damage. The effects of CBD on alcohol use and potential therapeutic implications

How to cite this article: Kessler FH, von Diemen L, Ornell F, Sordi AO. Cannabidiol and mental health: possibilities, uncertainties, and controversies for addiction treatment. Braz J Psychiatry. 2021;43:455-457. http://dx.doi.org/10.1590/1516-4446-2021-1838 
for alcohol use disorder are still unclear. ${ }^{12}$ There is also preliminary evidence for the treatment of opioids, psychostimulants, and other drugs. ${ }^{13,14}$ Also, observational studies suggest that CBD may reduce crack-cocaine addiction problems, such as withdrawal symptoms, craving, impulsivity, and paranoia. ${ }^{13}$

Recently, two very well-designed randomized, placebocontrolled trials failed to prove that CBD could decrease craving in individuals addicted to crack cocaine. ${ }^{15,16}$ Meneses-Gaya et al. published an interesting and original clinical trial, "Cannabidiol for the treatment of crackcocaine craving: an exploratory double-blind study," in the Brazilian Journal of Psychiatry. ${ }^{16}$ The authors emphasized that cannabinoids have been previously highlighted in uncontrolled observations as a strategy for reducing cocaine consumption and cravings, suggesting that these compounds may have a crucial clinical therapeutic role in treating crack-cocaine dependence. The sample size was small $(n=31)$, time since last use was heterogeneous, the CBD group seemed to have a more severe addiction profile, and craving was the primary outcome. All these limitations may have impacted the effect of CBD. Even though results were not satisfactory, this is one of the first relevant Brazilian studies in the addiction field concerning this molecule. ${ }^{16}$ Another study by Mongeau-Perusse et al. was conducted in Canada, with a larger sample size $(n=78)$, a higher dose of CBD (800 mg), and inpatient and outpatient phases. The authors found no improvement of $\mathrm{CBD}$ in reducing craving, days to relapse, days of cocaine use, or any other outcome. ${ }^{15}$ Both trials were conducted with heavy crack-cocaine users. The authors pointed out that CBD may work better in less severe groups. Even considering all the limitations, these two trials did not endorse the preclinical findings. ${ }^{15,16}$

Unfortunately, CBD is not always treated as a pharmacological agent under investigation; its use may be confounded with the cannabis legalization agenda. It is important to highlight that the expression "medical cannabis" has been used for all products derived from Cannabis sativa (THC, CBD, synthetic cannabinoids, and the whole plant). ${ }^{17-19}$ In the scientific context, it is imperative to analyze the results of studies involving specific molecules or combinations thereof instead of the whole plant. ${ }^{18}$ The great variety of substances being tested with contradictory results has led to misunderstandings in the field. ${ }^{17}$ For instance, the results of FDA approval of CBD for treating refractory seizures in two rare genetic syndromes should not be extrapolated to other components of Cannabis, the whole plant, or different seizure types. Furthermore, evaluation of components of Cannabis as a treatment should follow the same rigorous process as for other medications, with randomized, placebo-controlled clinical trials, evaluation of adverse effects, and dose-ranging efficacy. ${ }^{19}$ In particular, the placebo effect needs to be evaluated; in the aforementioned study by MongeauPérusse et al., both the placebo and CBD arms experienced less crack-cocaine cravings, indicating the importance of the placebo effect. ${ }^{15}$

In short, the scientific literature concerning this topic is still very incipient and presents mixed results. Despite excitement with initial results, most studies concerning
CBD for the treatment of substance use disorder present some biases that must be taken into consideration: heterogeneity of subjects and CBD doses, small samples, difficulty in controlling for comorbidities, withdrawal symptoms, medications, and open-label designs are some examples. Moreover, studies with positive results are more likely to be published. At this point, whether isolated CBD is as effective as what has been reported in the media about medical marijuana is still unknown. Nevertheless, further scientific investments are warranted to clarify if CBD has potential as a new tool for treating addictive disorders and their mental health burden.

\section{Disclosure}

The authors report no conflicts of interest.

\section{References}

1 Zuardi AW, Crippa JA, Hallak JE. [Cannabis sativa: the plant that can induce unwanted effects and also treat them]. Braz J Psychiatry. 2010;32 Suppl 1:S1-2.

2 Sarris J, Sinclair J, Karamacoska D, Davidson M, Firth J. Medicinal cannabis for psychiatric disorders: a clinically-focused systematic review. BMC Psychiatry. 2020;20:24.

3 Zuardi AW. History of cannabis as a medicine: a review. Braz J Psychiatry. 2006;28:153-7.

4 Köguel CC, López-Pelayo H, Balcells-Olivero MM, Colom J, Gual A. Psychoactive constituents of cannabis and their clinical implications: a systematic review. Adicciones. 2018;30:140-51.

5 Crippa JA, Guimarães FS, Campos AC, Zuardi AW. Translational investigation of the therapeutic potential of cannabidiol (CBD): toward a new age. Front Immunol. 2018;9:2009.

6 Pauli CS, Conroy M, Vanden Heuvel BD, Park SH. Cannabidiol drugs clinical trial outcomes and adverse effects. Front Pharmacol. 2020;11:63.

7 White CM. A review of human studies assessing cannabidiol's (CBD) therapeutic actions and potential. J Clin Pharmacol. 2019;59:923-34.

8 VanDolah HJ, Bauer BA, Mauck KF. Clinicians' Guide to cannabidiol and hemp oils. Mayo Clin Proc. 2019;94:1840-51.

9 Linares IM, Zuardi AW, Pereira LC, Queiroz RH, Mechoulam R, Guimarães FS, et al. Cannabidiol presents an inverted U-shaped dose-response curve in a simulated public speaking test. Braz $\mathrm{J}$ Psychiatry. 2019;41:9-14.

10 Bonaccorso S, Ricciardi A, Zangani C, Chiappini S, Schifano F. Cannabidiol (CBD) use in psychiatric disorders: a systematic review. Neurotoxicology. 2019;74:282-98.

11 Lee JL, Bertoglio LJ, Guimarães FS, Stevenson CW. Cannabidiol regulation of emotion and emotional memory processing: relevance for treating anxiety-related and substance abuse disorders. $\mathrm{Br} \mathrm{J}$ Pharmacol. 2017;174:3242-56.

12 Nona CN, Hendershot CS, Le Foll B. Effects of cannabidiol on alcohol-related outcomes: a review of preclinical and human research. Exp Clin Psychopharmacol. 2019;27:359-69.

13 Calpe-López C, García-Pardo MP, Aguilar MA. Cannabidiol treatment might promote resilience to cocaine and methamphetamine use disorders: a review of possible mechanisms. Molecules. 2019;24:2583.

14 Prud'homme M, Cata R, Jutras-Aswad D. Cannabidiol as an intervention for addictive behaviors: a systematic review of the evidence. Subst Abuse. 2015;9:33-8.

15 Mongeau-Pérusse V, Brissette S, Bruneau J, Conrod P, Dubreucq S, Gazil G, et al. Cannabidiol as a treatment for craving and relapse in individuals with cocaine use disorder: a randomized placebocontrolled trial. Addiction. 2021 Jan 19. doi: 10.1111/add.15417. Online ahead of print.

16 Meneses-Gaya C, Crippa JA, Hallak JE, Miguel AQ, Laranjeira R, Bressan RA, et al. Cannabidiol for the treatment of crack-cocaine craving: an exploratory double-blind study. Braz J Psychiatry. 2021;43:467-76. 
17 Pratt M, Stevens A, Thuku M, Butler C, Skidmore B, Wieland LS, et al. Benefits and harms of medical cannabis: a scoping review of systematic reviews. Syst Rev. 2019;8:320.

18 Lafaye G, Karila L, Blecha L, Benyamina A. Cannabis, cannabinoids, and health. Dialogues Clin Neurosci. 2017;19:309-16.
19 Bonn-Miller MO, EISohly MA, Loflin MJ, Chandra S, Vandrey R. Cannabis and cannabinoid drug development: evaluating botanical versus single molecule approaches. Int Rev Psychiatry. 2018; 30:277-84. 